\title{
COMMUNICATIONS.
}

\section{THE BLOOD-PRESSURE IN THE EYE AND ITS RELATION TO THE CHAMBER-PRESSURE.}

\author{
BY \\ Priestley Smith, \\ EMERITUS PROFESSOR OF OPHTHALMOLOGY, BIRMINGHAM UNIVERSITY.
}

A BETTER knowledge of the blood-pressure in the interior of the eye would help towards the solution of several important problems. It would help us to understand how the fluid which fills the chambers is produced and removed, how its pressure is maintained, and how changes in this pressure come about. Especially we want to know the normal pressure in the capillaries of the ciliary processes. Current estimates differ. In a recent discussion on the subject, Professor Leonard $\mathrm{Hill}^{1}$ maintained that the blood-pressure in the processes is about equal to the chamber-pressure, while Professor Starling gave reason for holding it to be higher than the chamber-pressure by at least $30 \mathrm{~mm}$. $\mathrm{Hg}$. My own contention was that whereas the pressure in the veins at their points of exit from the eye is about equal to the chamber-pressure, that in the capillaries must, in accordance with physical laws, be considerably higher. Since that time, by means of experiments on the pressure of water flowing through branching tubes, I have endeavoured to obtain a clearer idea of the pressure-changes which occur in the blood-stream as it passes through the eye.

The blood in the intra-ocular vessels, unlike the fluid in the chambers, moves with considerable velocity and consequently sufiers a loss of pressure between the points where it enters and leaves the eye. Under normal conditions its entrance-pressure is said to be about $100 \mathrm{~mm}$. Hg. Its exit-pressure cannot be far from $25 \mathrm{~mm}$. Hg. The evidence on which these estimates are based will be considered later; for our immediate purpose they may pass. We have then a fall of pressure of something like $75 \mathrm{~mm}$. Hg. between the entrance and the exit points. How is this fall distributed ? Does it take place chiefly on the arterial side of the capillaries, or on the venous side, or in the capillaries themselves? The present article approaches the question from the physical side, and deals firstly with the principles according to which a mass of liquid flowing through a tubular system loses pressure as it moves along.

To realize these principles effectively one needs the aid of practical demonstration - at least that is my own case. Hence the experiments. The manipulation was done for me by an assistant. We 
experimented first with tubes of the simplest form, and then modified the arrangement step by step so as to arrive ultimately at a branching system comparable in some degree with arteries, capillaries and veins. Many unforeseen difficulties arose, and the problem proved much more intricate than was anticipated. Before describing the experiments it will be well to remind the reader of certain fundamental facts.

A mass of liquid flowing through a tube exhibits energy in two forms; kinetic or employed energy in the movement of the liquid; potential or unemployed energy in its pressure against the wall of the tube. We are here concerned chiefly with lateral pressure. This can be measured at any point by making an aperture in the wall of the tube, connecting a vertical tube therewith, the connection being strictly at right angles with the wall of the tube, and noting the height to which the liquid rises. Placing a number of such tubes at selected points, and drawing a line through the summits of the columns so obtained, we get a pressure-line which shows in a graphic way how the lateral pressure of the stream varies in different parts of its course.

The lateral pressure at any specified point is a measure of the resistance encountered by the stream between that point and the distal end of the tube. At the proximal end of the tube the pressure is at its maximum, for here the whole of the resistance lies ahead; at the distal end it is nil, for here resistance ceases; between the two it diminishes progressively, for the unemployed energy which it represents is progressively expended in overcoming the resistence-being converted into heat. Hence in the case of any given tube, if the resistance be greater in one region of the tube than elsewhere, the pressure-line over that region will slope more steeply than elsewhere.

The resistance encountered in the tube depends on friction in the liquid itself, for the particles in the axis of the stream move with greater velocity than those nearer to the outer surface. The outer surface of the liquid, provided it wets the tube-wall, is practically stationary, so that there is no friction between the liquid and the wall itself. In a given tube, the resistance increases when the velocity of the stream increases; it is also increased by raising the viscosity of the liquid or by lowering its temperature.

Figure 1 shows the general arrangement of the apparatus employed in our experiments. The charts show the pressure-lines obtained. They represent the lateral pressure of water at a temperature of $60^{\circ}-65^{\circ} \mathrm{F}$. flowing through small glass tubes arranged in various ways. They are true, be it noted, only as regards the points showing actual measurements; the straight connecting lines are arbitrary. Had measurements been taken at intermediate points, all the deflected pressure-lines would have been somewhat different in shape. Had 
such points been infinitely numerous, sharp angles and straight connecting lines would have been absent; when not straight from end to end the whole line would have been sinuous, for changes of resistance do not take place abruptly-they tend to spread. But angular lines suffice for the present purpose.

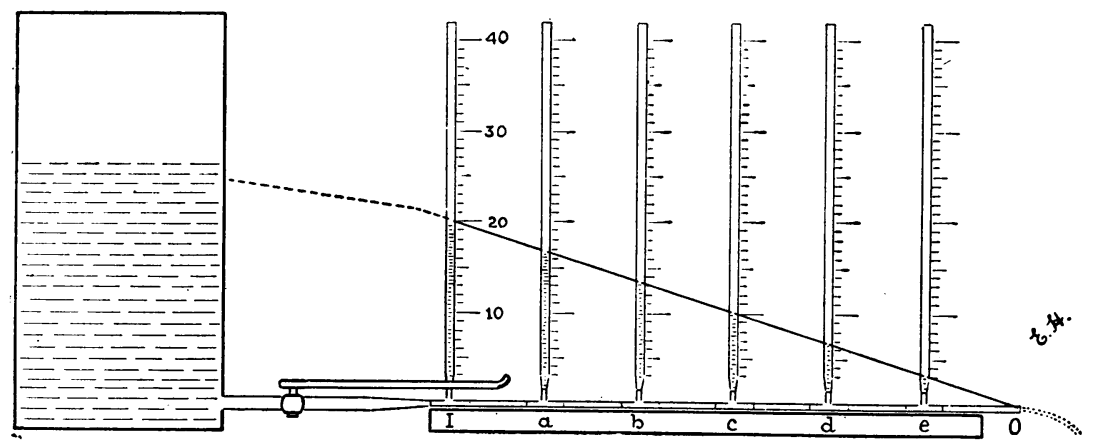

FIG. 1.

The water in the cistern, by its raised position, provides the energy that drives the stream through the horizontal tube. The tap admitting the water to the horizontal tube has a long 'handle, so that the rate of flow, and therewith the pressure at the point $I$, can be adjusted to a nicety.

The horizontal tube measures $60 \mathrm{~cm}$. from $I$ to $O$. $I$ stands for inlet, $O$ for outlet, and $c$ is midway between the two. The vertical tubes show the lateral pressure of the water at the points over which they stand; they are graduated in millimetres, although centimetres only are shown in the drawing; they are large enough to obviate material error from capillarity. The tubes and systems to be tested are placed between $I$ and $O$. They are built of segments connected end to end by short pieces of rubber tube. The vertical tubes are connected with the horizontal by means of $T$-pieces. Branching tubes are built by means of $\mathrm{Y}$-pieces. In the making of the tubes, narrowing of the lumen at the points of branching was avoided-it is a source of error easily overiooked.

In the case of symmetrical tubes the readings were controlled by repeating each experiment after reversing the position of the tube, the proximal end in the first trial becoming the distal in the second; where differences were found the average was taken ; the differences were small.

The readings were plotted on divided paper and the charts so obtained were reduced by photography with omission of the subdivisions. At the foot of each chart is shown the tube to which it refers-drawn somewhat too thick in proportion to its length. The larger numerals below the tube show the ratio of the cross-sections at the several points, and in the case of double and quadruple tubes they refer to the total cross-section. The -smaller figures show the diameters of the single tubes.

Details of tubes chiefly employed.

\begin{tabular}{|c|c|c|c|c|c|c|c|}
\hline Diameter & $\mathrm{mm}$. & 1.23 & 1.73 & 2.45 & 3.46 & 4.9 & \\
\hline of diameter & $\cdots$ & 1.5 & 3 & 6 & 12 & 24 & 48 \\
\hline Cross-section & ... sq. mm. & 1.2 & 2.4 & 4.8 & 9.6 & 19.2 & \\
\hline Ratio of cross-sectic & ons & 0.5 & 1 & 2 & 4 & 8 & 16 \\
\hline
\end{tabular}

In our earlier experiments we worked with a uniform "head" of water in the cistern, viz. $30 \mathrm{c} . \mathrm{m}$. This proved inconvenient. The pressure at the point $I$ is in every case lower than the cisternpressure, for energy is spent in giving motion to the stream at its 
exit from the cistern, and in overcoming resistance in the tap and nozzle; moreover, the difference between the pressure at $I$ and that in the cistern differs in different tubes according to the velocity of the flow; hence a uniform cistern-pressure gives various pressures at $I$, and the pressure-lines of different tubes are not easily compared.

Later we worked with a uniform pressure at $I, v i z .20 \mathrm{~cm}$. This was obtained, irrespective of the precise amount of pressure in the cistern, by careful adjustment of the tap. The pressure-lines so obtained all start at the same height on the charts and are therefore easily compared. A comparable condition in the vascular system would be a uniform blood-pressure at the commencement of the aorta.

Tube A (Fig. 2) is straight and of uniform diameter-the simplest possible form. The water moves through it with uniform velocity and meets with the same amount of resistance in each unit of distance travelled. Energy is therefore uniformly expended, lateral pressure falls evenly, and the pressure-line is straight." A pressure of $20 \mathrm{~cm}$. at $I$ gave the upper line seen in the chart, a pressure of $10 \mathrm{~cm}$. gave the lower; in each case the pressure at the half-way point equals the mean of the end-pressures.

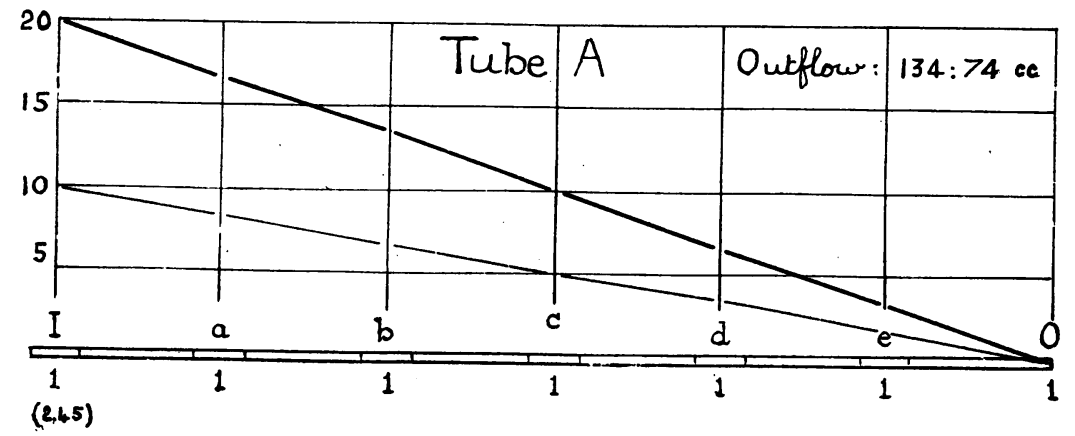

FIG. 2.

Tube B (Fig. 3), resembles the foregoing, except that it enlarges in the middle region. The cross-section at $c$ is twice that at $b$; the velocity at $c$ is therefore half that at $b$. The pressure-line changes with the change of velocity; it is pushed upwards over the middle region of the tube; the half-way pressure is greater than the mean of the end-pressures.

In contrast with the foregoing, Tube $\mathrm{C}$ (Fig. 3) diminishes in the middle. The cross-section is reduced here by one-half and the velocity is thereby doubled. The pressure-line is deflected downwards in the middle region; the half-way pressure is below the mean.

Tubes with greater enlargement in the middle region gave greater 
upward displacement (see for example Tube L), and tubes with more contraction in the middle gave more downward displacement; but the foregoing examples are sufficient. They illustrate a principle :-In a symmetrically-shaped horizontal tube of uniform diameter the half-way pressure equals the mean of the end-pressures; in one which enlarges in the middle the half-way pressure is greater than the mean; in one which diminishes in the middle it is less than the mean.

Now, in one respect the vascular system of the body resembles a tube which enlarges in the middle: its aggregate cross-section enlarges progressively up to the half-way point and diminishes progressively beyond that point. But it is not a single tube and its

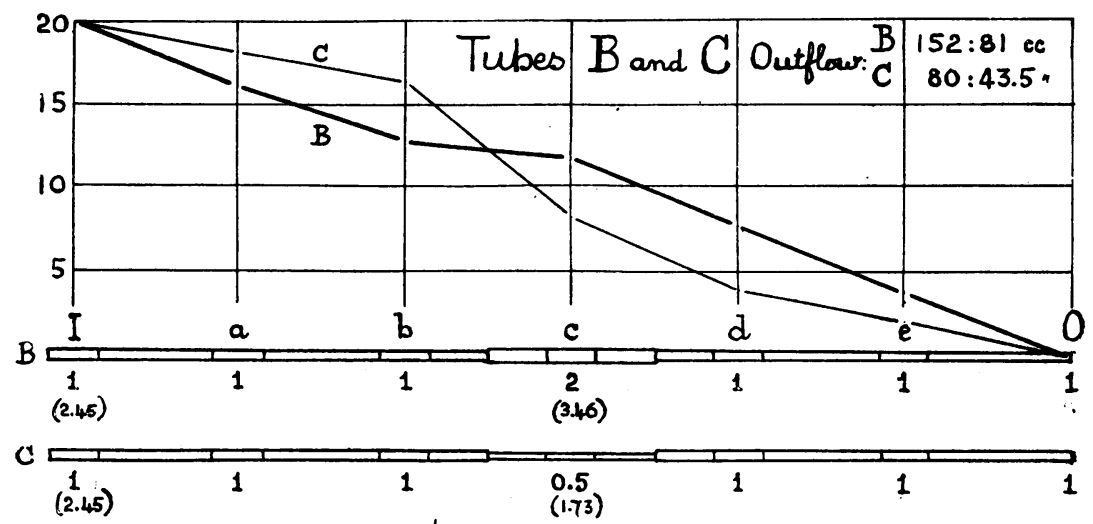

FIG. 3.

proximal and distal halves are not symmetrical, and these differences must be taken into account before any inference relating to bloodpressure can be based on the principle just stated. Let us first observe the influence of branching in symmetrical systems.

In Tube D (Fig. 4) the two branches are of equal size, and their aggregate cross-section equals that of the trunk. Consequently each

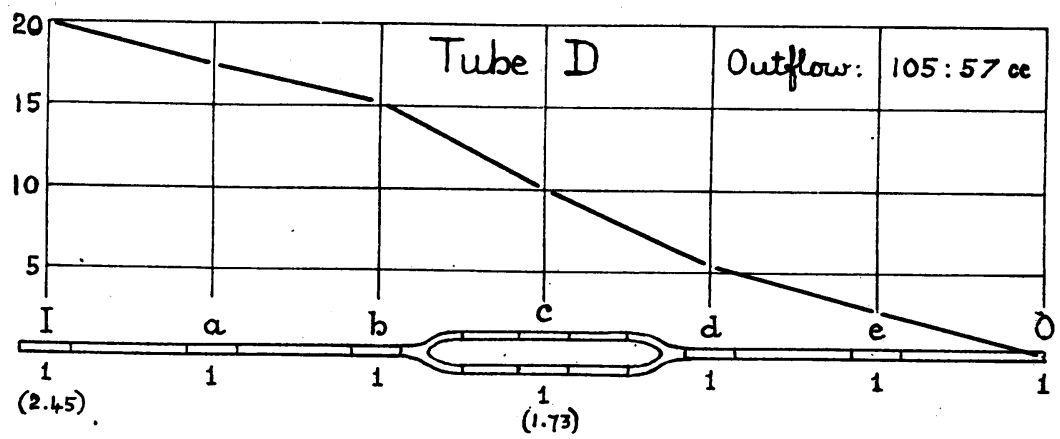

FIG. 4. 
branch transmits one-half of the stream at the same rate as that at which the trunk transmits the whole : at $c$ the velocity is the same as at $b$ and $d$. As in the case of the single tube with uniform velocity throughout (Tube $A$ ) the half-way pressure equals the mean of the end-pressures, although here the resistance is not uniform throughout, but greater in the middle region, as shown by the varying slope of the pressure-line.

In Tube E (Fig. 5) each branch alone is equal to the trunk. At $c$ therefore the total cross-section is double that at $b$, and the velocity is reduced to one half. As in the corresponding single tube $\mathrm{B}$, the pressure-line is deflected upwards from its straight course ; the pressure at the half-way point is above the mean.

In Tube F (Fig. 5), on the contrary, the total cross-section at $c$ is half that at $b$, and the velocity is doubled. The pressure-line is deflected downwards; the pressure at $c$ is below the mean.

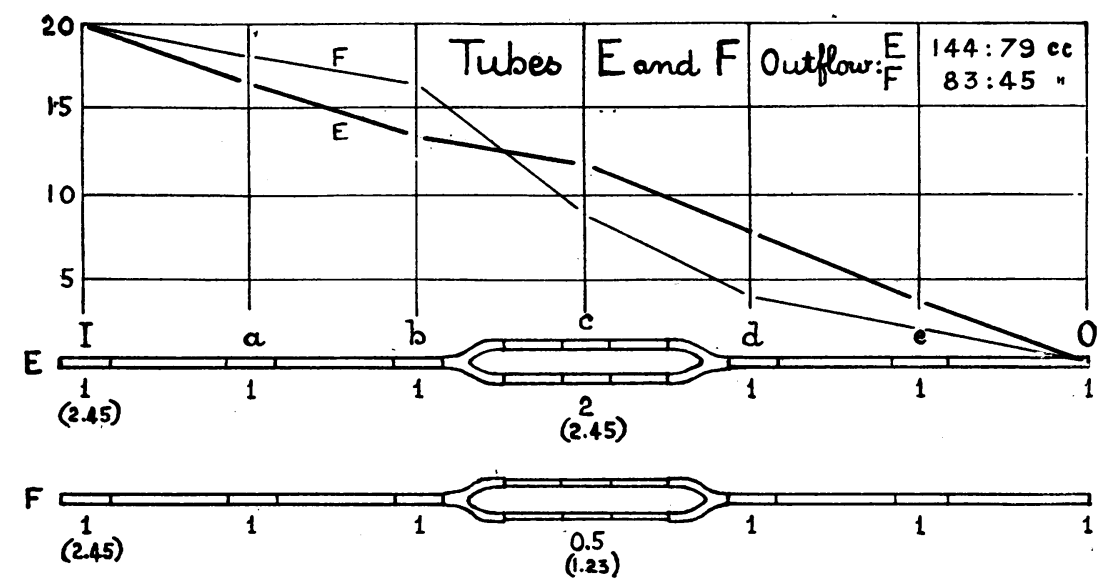

FIG. 5.

Tubes $\mathrm{G}$ and $\mathrm{H}$ (Figs. 6 and 7) carry the demonstration one step further. In $\mathrm{G}$ the branches arising at each bifurcation are together equal to the trunk from which they spring; the total cross-section at $c$ is therefore equal to that at $b$, and the velocity is uniform throughout the whole system, as in Tubes A and D. Here again, with uniform velocity, we have the half-way pressure equal to the mean in spite of the repeated branching.

In Tube $\mathrm{H}$, on the other hand, the branches are individually equal to the trunk; the total cross-section at $c$ is therefore four times that at $b$ and the velocity is reduced to one-fourth. As before, with lowered velocity at the half-way point the half-way pressure is above the mean.

But neither of these branching tubes represents the method of 
branching which is usual in the case of blood-vessels. When an artery bifurcates the branches, as a rule, are neither collectively nor individually equal to the trunk; they are formed on an intermediate plan: together they are larger, singly they are smaller, than the

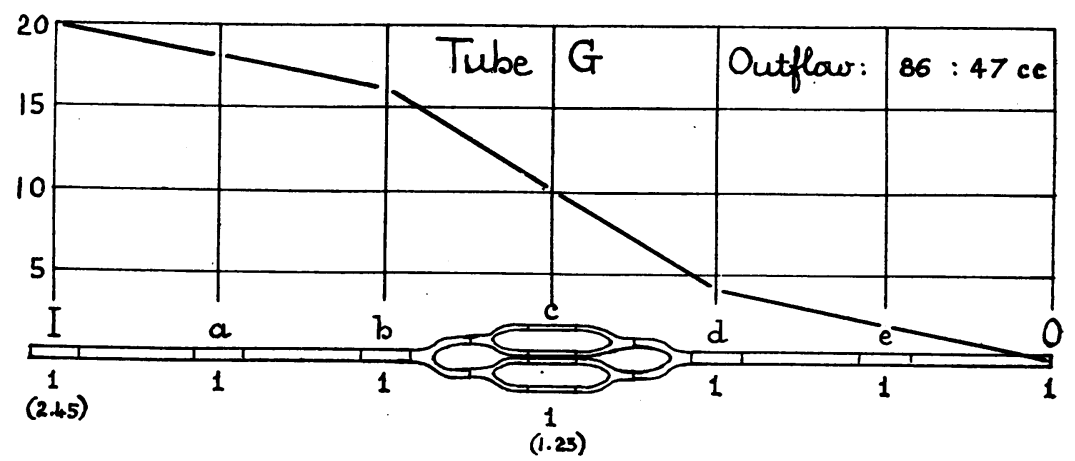

FIG. 6.

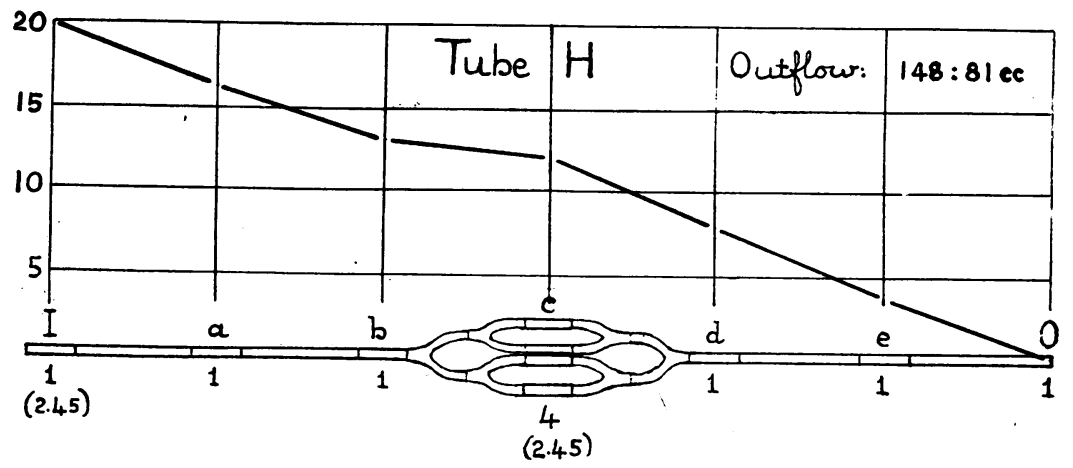

FIG. 7.

trunk. Tube J (Fig. 8) is built on such a plan. The total crosssection increases at each bifurcation, but less rapidly than in Tube $\mathrm{H}$; it doubles in two bifurcations; at $c$ it is twice as great as at $b$. The pressure-line is deflected upwards; the half-way pressure is above the mean, but less so than in the case of Tube $\mathrm{H}$ where the enlargement occurred more rapidly and was greater.

The experiment was carried further yet. Wishing to obtain conditions more nearly comparable with the vascular system, namely, a multitude of small channels with great extension of friction-surface as well as enlargement of cross-section in the middle region, and finding that branching tubes of still smaller size could not be made with the required accuracy, we proceeded in another way.

Tube K (Fig. 9) is identical with Tube J, save as regards the four 
central T-pieces. These are larger than in Tube J, and are packed with capillary tubes. Instead of 4 tubes at $c$, we have now 36, and about twice as many small angular spaces between the tubes. The aggregate lumen at $c$ is to that at $b$ about as 6 to 1 . The pressure-line differs little from that of Tube J; the half-way pressure is above the mean.

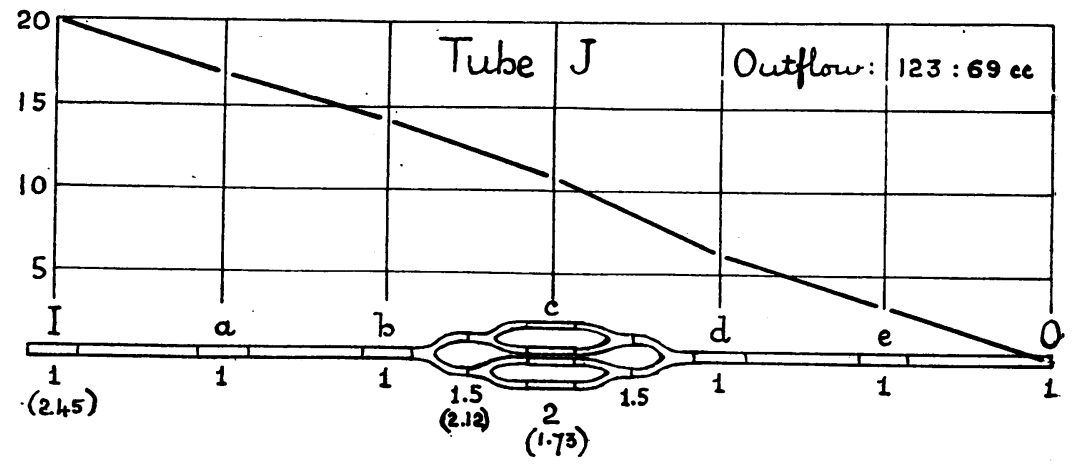

FIG. 8.

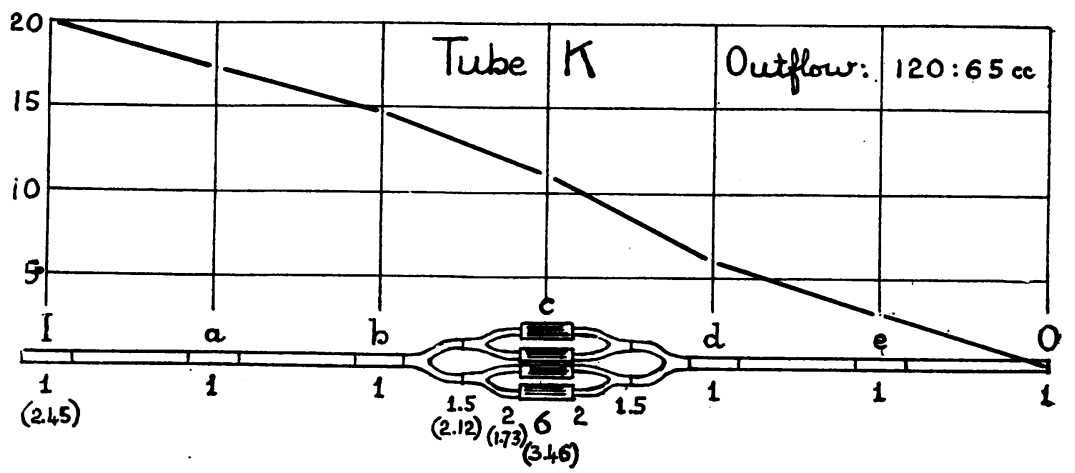

FIG. 9.

The capillary tubes were $20 \mathrm{~mm}$. long, and shorter than the containing tubes by $5 \mathrm{~mm}$. at each end, so that the water had easy access to them all. Each containing tube held 9 capillaries; the average diameter of the capillaries was about $0.9 \mathrm{~mm}$. outside, 0.75 inside.

Evidently what we found to be true for symmetrical single tubes is true for symmetrical branching tubes also:-If the aggregate cross section of the branches at the half-wav point of a symmetrical branching tubular system is greater than the cross-section at the ends, the half-way pressure is greater than the mean of the end pressures. 
Now, the blood-stream enlarges greatly as it travels from the aorta to the capillaries-roughly about 600 times. We may therefore infer that if the vascular system were strictly symmetrical, i.e. if the veins were an exact counterpart of the arteries, and if the whole system were horizontal, the pressure in the capillaries would be greater than the mean of the pressures in the main trunks.

How much greater would it be? Consider the excess in some of the foregoing examples. The end-pressures in every case are 20 $\mathrm{cm}$. and 0 , the mean is therefore always $10 \mathrm{~cm}$. In Tubes $\mathrm{B}$ and $\mathrm{E}$ the half-way pressure exceeds the mean by 1.8 and $1.7 \mathrm{~cm}$. respectively, i.e. by 9 and 8.5 per cent. of the total fall. In Tube $\mathrm{H}$ it exceeds the mean by 10 per cent. of the total fall. But in these examples the cross-section in the middle is only twice as great as that at the ends. With greater enlargement we obtain greater

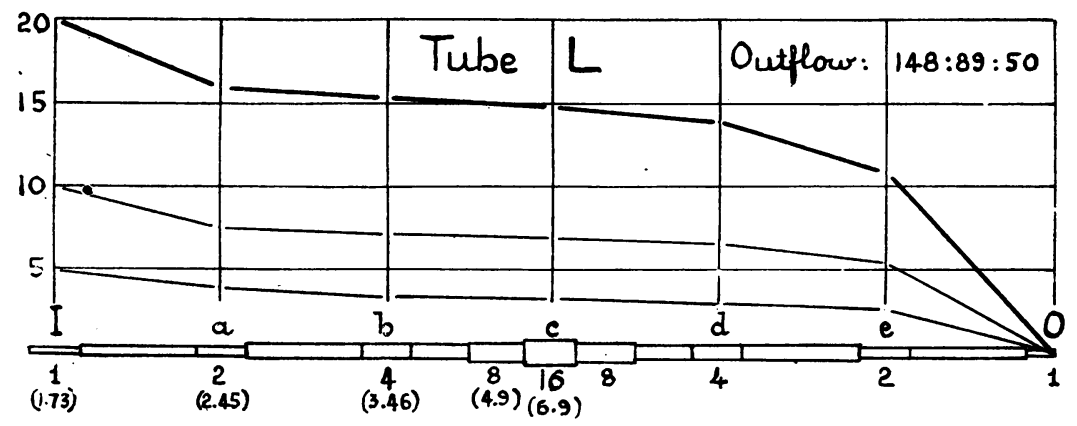

FIG. 10.

excess; for example, in Tube L (Fig. 10) where the cross-sections at $c$ and $I$ are as 16 to 1 , the half-way pressure exceeds the mean by no less than 24 per cent. of the total fall. Now, in the vascular system of the body the ratio of enlargement in the middle region is very much greater still. May we, then, infer that if arteries and veins were alike in size and distribution, and the whole system were horizontal, the pressure in the capillaries would greatly exceed the mean of the pressures in the main trunks?

No. The results obtained so far do not justify this inference, for the amount of the excess in question varies, not only with the amount of enlargement in the middle region of the system, but also with the velocity and steadiness of the stream, and with the distribution of the resistance which has to be overcome. These factors must be taken into account, and the artificial streams must be compared with the blood-stream in these respects, before any inference of the kind can be safely drawn.

The influence of velocity on the excess of pressure at the half-way point of a given tube is seen when we alter the velocity. Changes 
of velocity can be measured by measuring the amounts of liquid escaping from the tube in equal periods of time, for in a given tube the outflow is directly proportional to the velocity. In our experiments the outflow during periods of 60 seconds was measured by means of a tall measure-glass graduated in c.c., and the seconds hand of a watch. The results are shown on the charts.

Tube L, for example, was tested under three different pressures, viz. 20,10 , and $5 \mathrm{c.m}$. Lowering the pressure lowered the velocity of the stream, as shown by the diminished outflow-148, 89, 50 c.c. in 60 seconds, and at the same time the excess of pressure at the half-way point fell from 4.8 to 1.9 and $0.6 \mathrm{~cm}$., i.e. from 24 to 19 and 12 per cent. of the total fall of the pressure-line. Here are the figures tabulated:

Tube L

Under pressure $20 \mathrm{c.m}$.
Actual excess in $\mathrm{c} . \mathrm{m}$.
Equivalent percentage of total fall.
Outflow in c.c.

1.9

0.6
24
... 12

$\ldots$

$\cdots$

,

5 c.m.

In the self-same tube then the excess of pressure at the half-way point was smaller in relation to the total fall when the water flowed slowly than when it flowed fast.

Now the question of turbulence. In a turbulent stream energy is expended in the formation of eddies, whirls, and back-currents ; in a smoothly-flowing stream no energy is wasted in this way. It was necessary, therefore, to ascertain whether our artificial streams were smooth or turbulent, and to compare them with the bloodstream in this respect.

In the case of a steady stream the velocity is directly proportional to the pressure, e.g., if we double the pressure we double the velocity, and thereby double the outflow per unit of time. In a turbulent stream the velocity is not proportional to the pressure; when we double the pressure we fail to double the outflow, for more of the energy is wasted in the formation of eddies. Hence, to ascertain the character of a stream in this respect, we have only to measure the outflow under two known pressures, say 20 and $10 \mathrm{c.m}$. If we fail to halve the outflow when we halve the pressure, we know that the stream is more or less disturbed by eddies. When the need for this control became evident, we rebuilt Tubes A to L, and in each case measured the outflow per 60 seconds under the pressures named. In not one of these cases, as the charts will show, were the outflows proportional to the pressures employed. We therefore modified the apparatus so as to obtain steady streams. Three courses were open to us.

The steadiness or unsteadiness of such streams is determined by three factors : the size of the tube, the velocity of the stream, and 
the viscosity of the liquid. A given liquid will flow steadily through a given tube, provided the velocity be not too great; a given liquid will flow steadily at a given velocity provided the tube be not too large; in a given tube, at a given velocity, one liquid will flow steadily, whereas a less viscous one will be thrown into eddies. Steadiness demands that the result of these three factors shall not exceed a certain critical value. It was open to us, therefore, either to increase the viscosity of the liquid, to diminish the velocities by employing lower pressures, or to reduce the size of the tubes. We chose the latter course.

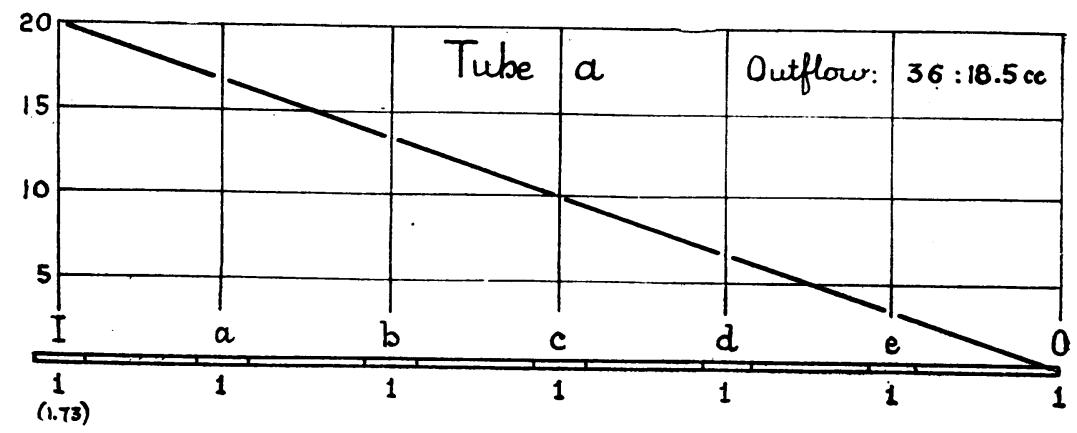

FIG. 11.

Tubes a, b, c (Figs. 11 and 12) were built to resemble the original Tubes A, B, C in all respects, except in the size of their crosssections ; these were smaller by one-half. The outflows from these smaller tubes were found to be very nearly proportional to the pressures employed; the streams may therefore be regarded as approximately steady. Here are the outflows in c.c. per minute from the larger and smaller tubes respectively :

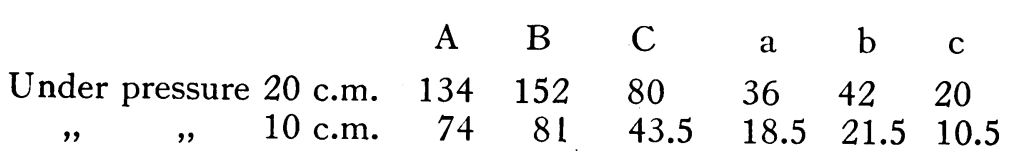

Now compare the pressure-lines of the larger and smaller tubes respectively. Tubes $\mathrm{A}$ and $\mathrm{a}$, although differing in size, give identical lines, for in each case the half-way pressure equals the mean of the end-pressures and there is no deflection. Tubes $B$ and $b$ give differing lines : in each case the line is deflected upwards in the middle region, but the deflection is smaller in the case of the smaller tube. Tubes $\mathrm{C}$ and $\mathrm{c}$ give downward deflections, and here again the smaller tube gives the smaller deflection. The following table 
shows the differences, plus or minus, between the half-way pressures and the mean-pressures in percentages of the total fall :

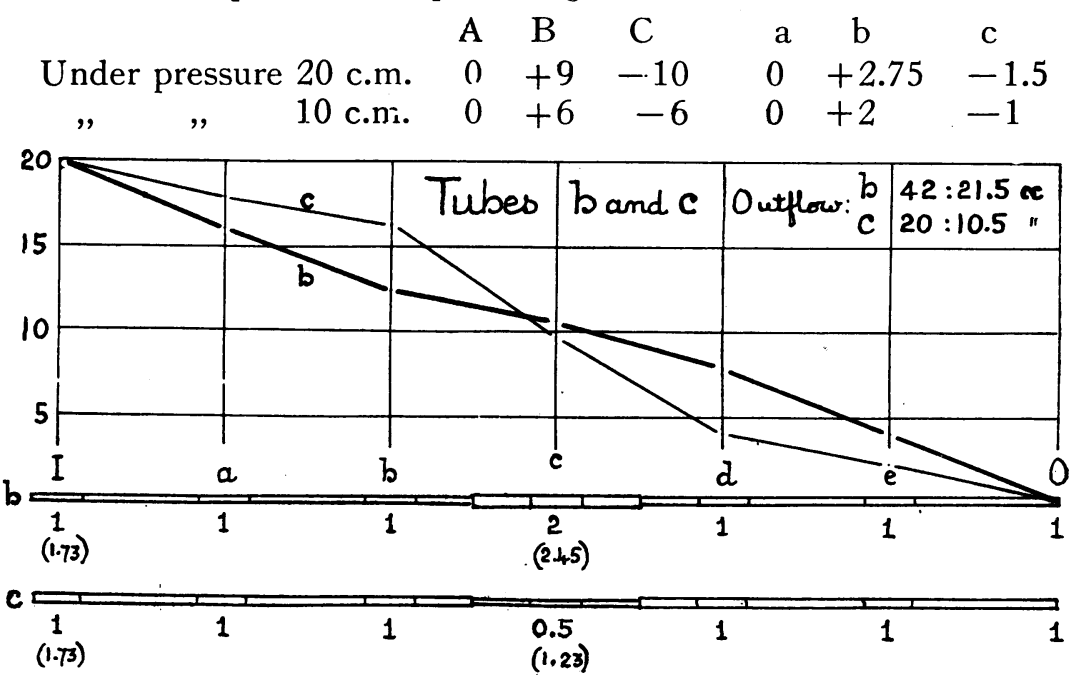

FIG. 12.

The smaller tubes, then, gave steadier streams and smaller deflections of the pressure-line. But it must not be assumed that the deflections were smaller only because the streams were steadier, for they were also slower. Lowering of velocity was associated with lessened deflection even in a stream which was practically steady. Thus in Tube $\mathrm{b}$ when the velocity was reduced approximately by one-half, the percentage-deflection fell from 2.75 to 2, and in Tube c, under the same circumstances it was reduced from -1.5 to -1 . (Velocity in $\mathrm{mm}$. per second is determined by dividing the outflow in cub. mm. per second by the cross-section in sq. mm.) Here are the end-velocities in $\mathrm{mm}$. per second in the two sets of tubes respectively :-

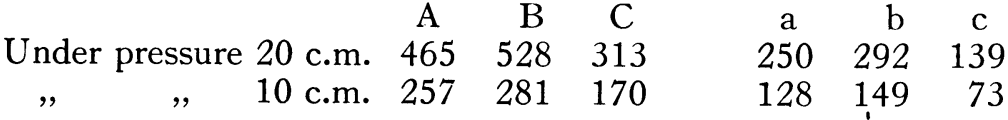

Again, tubular systems that are alike as regards cross-section may differ greatly as regards the amount and distribution of the resistance they offer to the liquid flowing through them, and this may modify the excess of pressure at the half-way point to a very large extent. In considering the following examples the reader will remember that he can compare the amounts of resistance in different regions of a given tube by observing the varying slope of the pressure-line: where the slope is steeper the resistance is greater.

Tubes $\mathrm{M} \mathrm{N}$ and $\mathrm{O}$ (Fig. 13) are alike as regards the total crosssections of the channels through which the water flows. In each 
case the cross-sections of the 5 segments of the tube are respectively as $\begin{array}{llllll}1 & 2 & 4 & 2 & 1\end{array}$. On the other hand, they differ as regards the amount and distribution of the resistance they offer to the stream.

Tube $M$ is fully open throughout, and the resistance as shown by the pressure-line (the thin line) is greatest in the end-segments, least in the middle.

Tube $\mathrm{N}$ is identical with the foregoing as regards the endsegments. The 3 middle segments, if left fully open, would have twice the cross-section of those of Tube $\mathrm{M}$; but they are packed

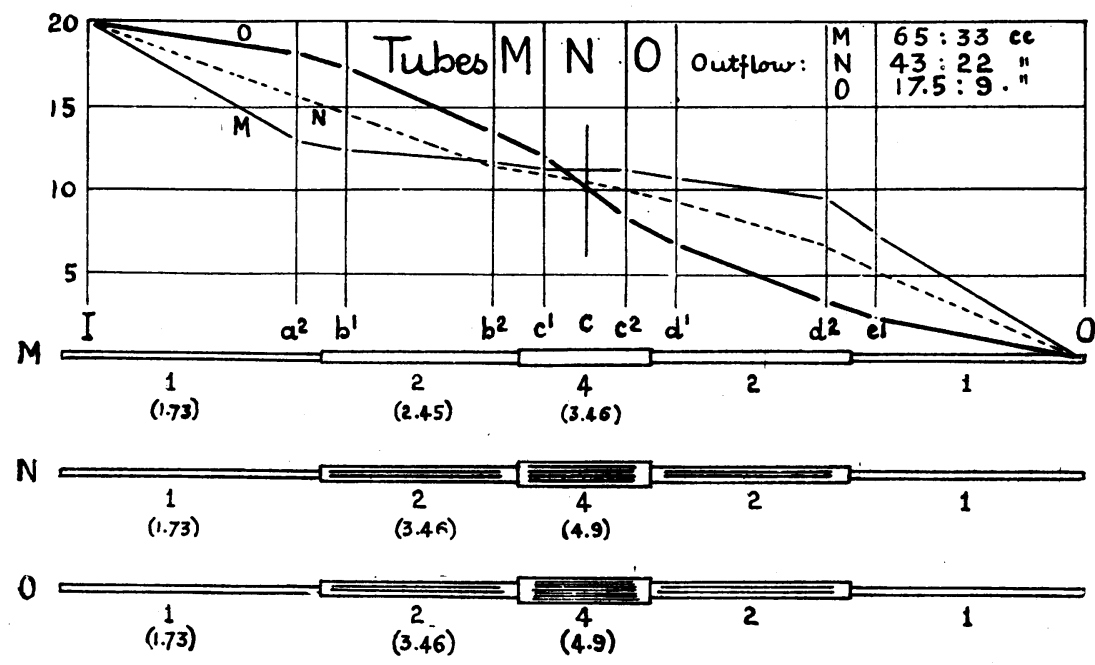

FIG. 13.

with straight wires in such a way as to reduce their effective lumen to equality with Tube M. The increased friction-surface given by the wires adds to the resistance in these segments of the tube, and the wires are so chosen as to number and diameter as to give nearly equal resistance throughout the whole length of the tube-as shown by the nearly uniform slope of the pressure-line (broken line).

Tube $\mathrm{O}$ is identical with Tube $\mathrm{N}$ as regards the containing tubes, but is packed with a larger number of smaller wires so chosen as to give the same effective lumen as that of Tubes $M$ and $N$, and at the same time a greater resistance in the middle than at the endsas shown by the pressure line (thick line).

Here then are three symmetrical systems all enlarging in the middle region, alike in their initial diameter and in the amount of their enlargement, but different as regards the amount and distribution of the resistance they offer to the stream. The point of interest is that while the half-way pressure exceeds the mean in each, the excess is smallest where the resistance in the middle region is 
greatest: under a pressure of $20 \mathrm{~cm}$. at $I$ the excess in the three tubes $\mathrm{M} \mathrm{N}$ and $\mathrm{O}$ was respectively $6.5,2.5$, and 0.75 per cent. of the total fall.

The difference is partly due, no doubt, to the lower velocities in the packed tubes: the velocities are proportionate to the outflows, and these were respectively 65,43 , and $17.5 \mathrm{c} . \mathrm{m}$. in 60 seconds. Is it wholly due to this? Apparently not. To test the point we subjected each tube to three different pressures viz. 40,20 and 10 $\mathrm{cm}$. and obtained the following results:-

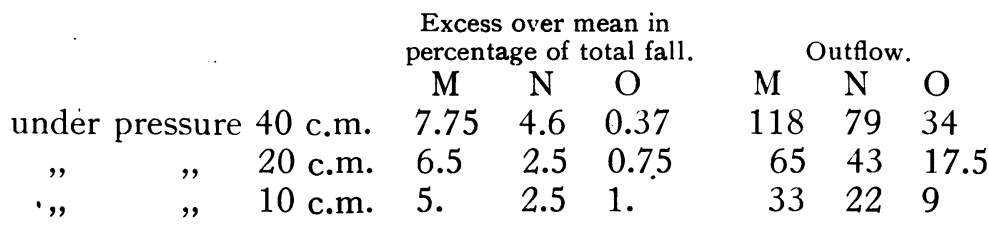

It will be seen that Tube $\mathrm{O}$ under a pressure of $40 \mathrm{c}$.m. gave approximately the same outflow (same velocity) as Tube $\mathrm{M}$ under $10 \mathrm{c} . \mathrm{m}$. The conditions in these tubes, therefore, were alike not only as regards total lumen but also as regards velocity, and yet the excess of pressure at the half-way point was in the one case only 0.37 per cent. of the total fall, while in the other it was 5.0 per cent. The amounts are small, and one cannot safely attach a precise value to the figures, but they appear to justify the following conclusion: A single tube and a branching system that are equal in aggregate cross-section, that enlarge equally in the middle region, and that transmit streams of equal velocity, do not give equal excess of pressure at the half-way point; the excess is smaller in the branching system. The large excess seen in the case of Tube $\mathrm{L}$ and others of that type is no criterion then for a minutely ramifying system.

Let us now turn to the blood-stream and consider it with regard to these same influences-first as to velocity and steadiness of flow, then as to distribution of resistance.

In certain regions the velocity of the blood-stream has been measured. In the arch of the aorta, it is probably about $300 \mathrm{~mm}$. per second. In the capillaries of different parts, it has been found to be from 0.2 to $0.8 \mathrm{~mm}$., say on the average $0.5 \mathrm{~mm}$. per second, although the variations are probably greater than those here stated. In blood-vessels comparable in size with our artificial tubes, therefore, the velocity is probably lower than it was in our experiments.

As regards turbulence, it is important to note that the natural channels are built with special adaptations for the minimising of resistance at the points of branching, and are therefore more favourable to steady flow than are our crudely-built artificial systems. Moreover, blood is more viscous than water. Certainly, therefore, 
the blood-streams are free from turbulence throughout the greater part of their course, and it seems probable, from certain experiments directed to the question, ${ }^{2}$ that under normal conditions they are so even in the larger vessels. Turbulence involves loss of energy, and Nature in her living mechanisms is chary of such waste.

The elasticity of the arteries, be it noted, is an important factor in the avoidance of turbulence, for the blood-stream is driven by intermittent force. In our experimental apparatus, where the driving-force is constant, the substitution of elastic for rigid tubes would give no advantage in this respect, for under unvarying pressure such tubes would assume fixed dimensions. The difference, therefore, does not invalidate comparison as regards the point in question.

Lastly, the distribution of the resistance. It is easy to prove that in the vascular system of the body the resistance per unit of distance travelled-taking the multiple streams as one-increases from the aorta to the capillaries, and that the increase is very large. Two opposing influences are here at work : the lowering of the velocity of the blood, as it moves from the aorta to the capillaries, tends to diminish the resistance; the multiplication of channels tends to increase it. Which influence preponderates? We cannot experiment on a vast multitude of minute channels like those through which the blood is driven, but we can measure resistances in various simpler types of branching tube, and so arrive at a principle applicable to the more complex.

To compare resistances in different regions of a tubular system we have only to measure the fall of the pressure-line over equal lengths of the tube in the specified regions: the resistances are proportional to the amounts of fall. Let us first take the case of a tube which enlarges simply by increase of diameter, not by branching.

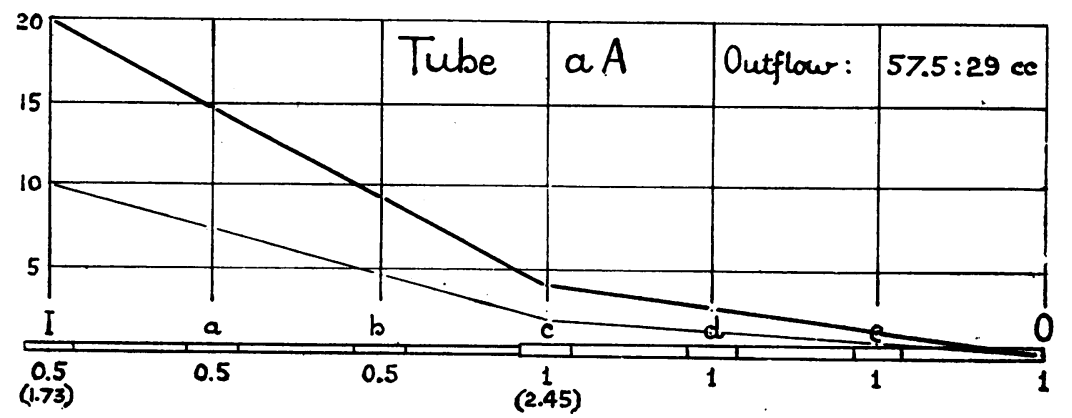

FIG. 14.

In Tube aA (Fig. 14) the cross-sections of the proximal and distal halves are as 1 to 2 . Over the proximal half the pressure-line falls from 20 to $4 \mathrm{~cm}$., over the distal half from 4 to 0 ; the falls are 
therefore as 4 to 1 , in other words, the distal fall is 20 per cent. of the total fall.

To realise the reason of this, note, firstly, that the two halves of the tube transmit the same quantity of water in a given time. Now,
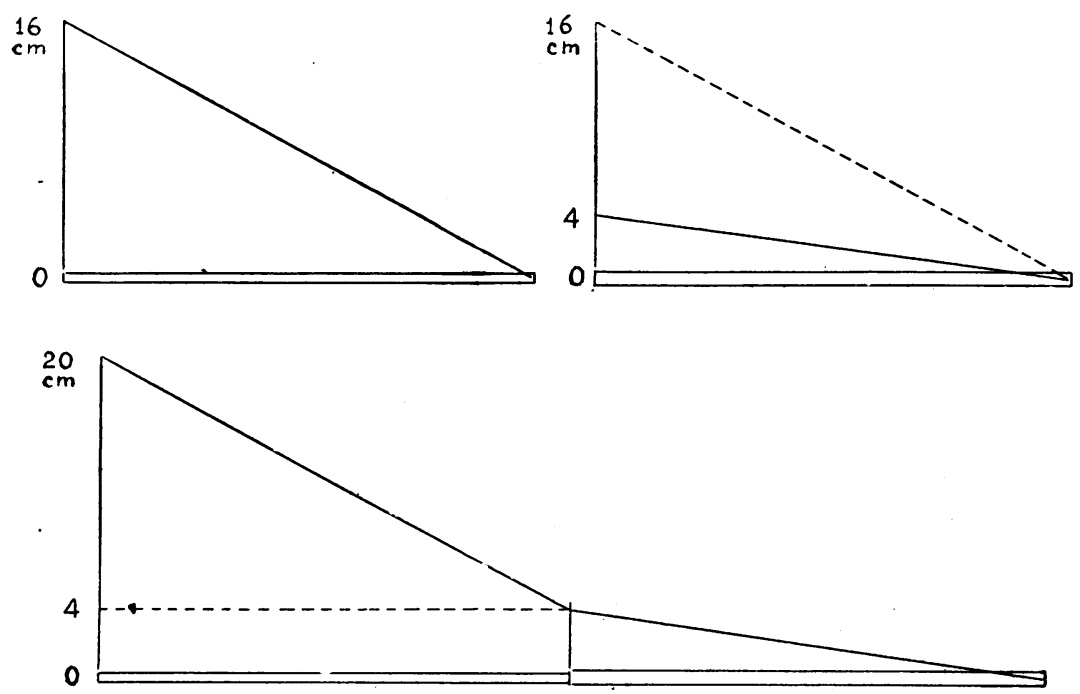

FIG. 15.

imagine the two halves to be disconnected and tested separately, each under an inlet pressure of $16 \mathrm{~cm}$. (Fig. 15). Their pressurelines would be identical, but the larger tube would transmit four times more water in a given time than the smaller, because it has double the capacity, and its stream has double the velocity. If the larger portion is to transmit the same quantity as the smaller, as it does in the entire tube, we must lower its inlet pressure from 16 to $4 \mathrm{~cm}$. And if this same rate of flow is to be maintained when the two portions are reunited, we must raise the inlet-pressure of the entire tube from 16 to $20 \mathrm{~cm}$. Then the stream, on reaching the half-way point, will still retain an unexpended lateral pressure of $4 \mathrm{~cm}$., and this will carry it through the second half of the tube at the required velocity.

Now turn to branching tubes of several types. For the sake of accuracy it is well to take the pressures at points not too near to the bifurcations: to measure equal lengths of trunk and branches and to exclude the disturbances occurring at the points of bifurcation.

Tube $\mathbf{P}$ (Fig. 16) consists of a trunk and 2 equal-sized branches; the total cross-section of the branches equals that of the trunk. Over region $I b$ the pressure falls $4.3 \mathrm{~cm}$; over $d O$ it falls $8.7 \mathrm{~cm}$.a ratio of approximately 1 to 2 . In Tubes Q R S (Figs. 17, 18, 19) 
measured in like manner, we find respectively ratios of approximately 1 to 1,1 to 0.5 , and 1 to 0.25 . (The ratios actually given by the readings were $1: 2 \cdot 02,1: 1^{\circ} 04,1: 0^{*} 47$, and $1: 0 \cdot 26$; there were, no

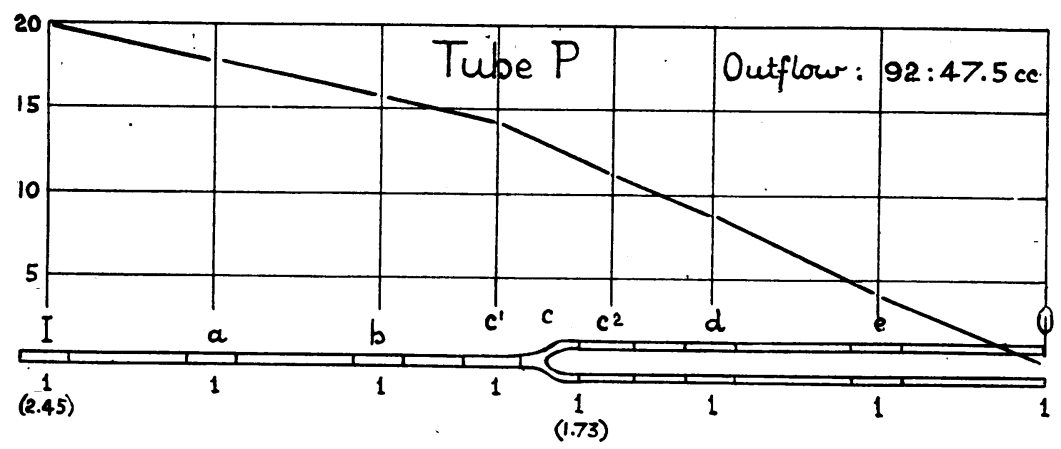

FIG. 16,

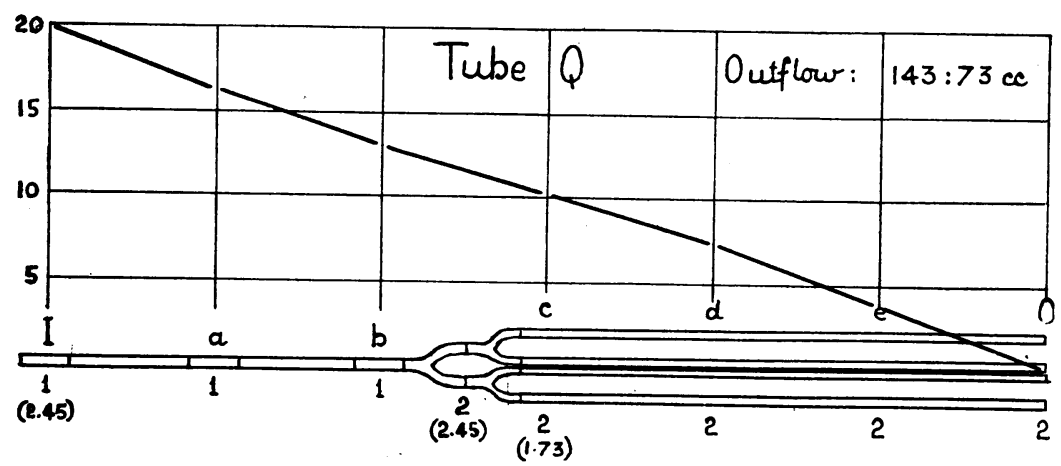

FIG. 17,

doubt, slight dimensional faults in the apparatus). These ratios represent the resistances respectively in equal lengths of trunk and branches, taking branches in the aggregate. From them the following rule may be deduced:-

To find the relative resistances in different regions of a tubular system in which companion branches are of equal size: Square the total cross-section in each region, and divide by the number of branches in that region; the products are inversely proportional to the resistances.

By means of this rule we can roughly calculate the relative resistances in equal lengths of the aorta and the capillary tract. Take the mean velocity in the arch of the aorta at $300 \mathrm{~mm}$. per second, and that in the capillaries at $0.5 \mathrm{~mm}$. per second ; then, since velocity and cross-section in different regions of a given tubular system are inversely proportional, the cross-section of the aorta is to 
that of the whole capillary tract as 0.5 to 300 , or as 1 to 600 . Again, take the cross-section of the aorta to be $500 \mathrm{sq} . \mathrm{mm}$.; then that of the capillaries will be $500 \times 600$, i.e., 300,000 sq. $\mathrm{mm}$. Vierordt ${ }^{3}$ puts it somewhat higher, but for our present purpose the exact figure is not important. Again, if we take the diameter of

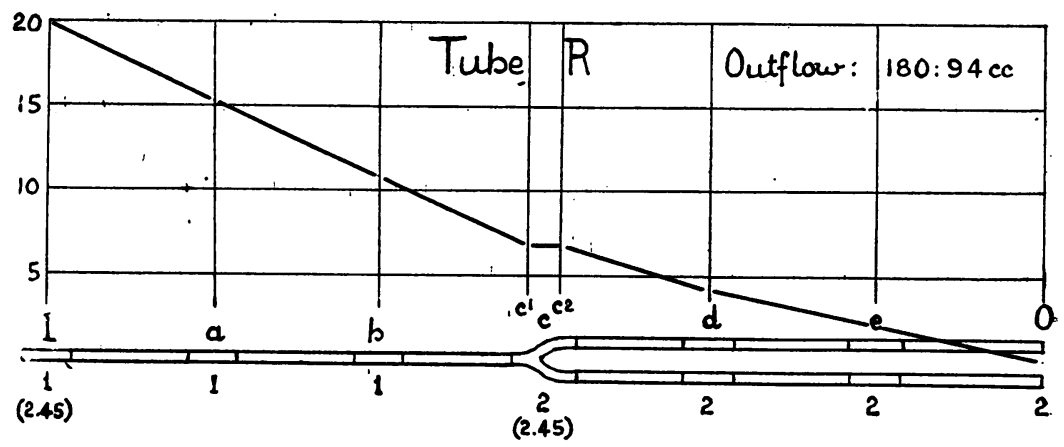

FIG, 18 .

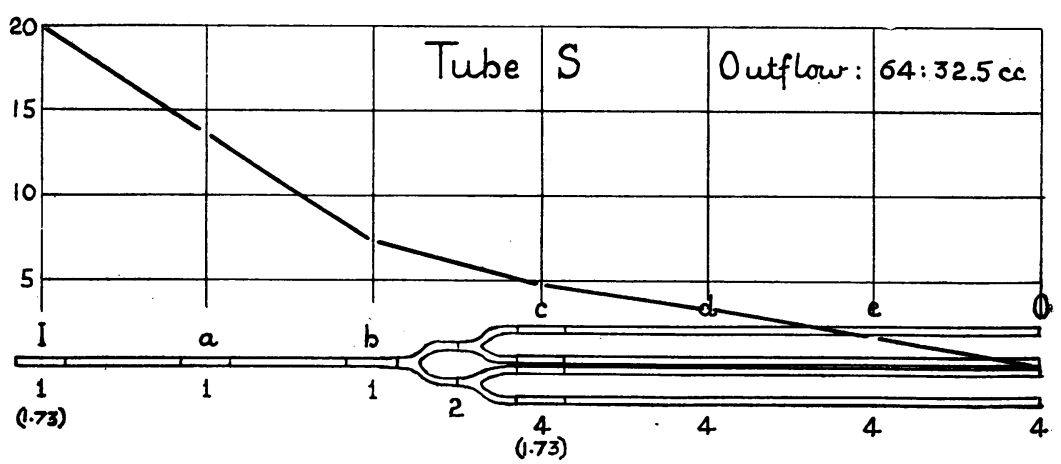

FIG. 19,

an average capillary to be $0.01 \mathrm{~mm}$.; its cross-section will be 0.00007854 sq. mm.; then dividing the total cross-section of the capillaries by this figure we find that the total number of the capillaries is four thousand million. These then are the data :-

Ratio of cross-sections Aorta.

Number of tubes ...

$$
\begin{array}{cccc}
\ldots & 1 & \ldots & 600 \\
\ldots & 1 & \ldots & 4,000,000,000
\end{array}
$$

and according to the rule just given, the resistances in the aorta and capillaries respectively will be inversely as 1 squared divided by 1 $(=1)$ to 600 squared divided by 4,000,000,000 (=approximately $0.0001)$; i.e., as 1 to 10,000 . The result is startling, but we must remember that the length of an average capillary is about $0.5 \mathrm{~mm}$., and that we are comparing the resistance in the whole of: 
the capillaries with that in an equal length $(0.5 \mathrm{~mm}$.) of the aorta. The resistance in $10 \mathrm{~cm}$. (4 inches) of the aorta would be to that in the capillary tract as 1 to 50 . The calculation may involve considerable error by reason of faulty data, but there can be no doubt that while the cross-section of the blood-stream increases greatly from aorta to capillaries, the resistance it has to overcome increases in a much higher ratio still.

It appears then, that as regards the influences in question-velocity, steadiness of flow, and distribution of resistance-the vascular system of the body is more nearly comparable with tube 0 than with any other in our experimental series; hence we may infer that if the vascular system were symmetrical and horizontal the pressure in the capillaries would exceed the mean of the pressures in the main trunks by a very small percentage only of the total fall.

Under the supposed conditions the pressure-line of the bloodstream would have the general character of the upper line in Figure 24. At the proximal end its gradient would at first be indistinguishable from a horizontal line; gradually, through progressive multiplication of the channels, it would slope more and more, and in the middle region it would be very steep. At the half-way point it would indicate a pressure slightly above the mean of the end-pressures. Beyond that point it would become progressively less and less steep, and near to the distal end would again be apparently horizontal.

But the vascular system of the body is not symmetrical in its proximal and distal halves. The veins-excepting the pulmonary veins-have everywhere a larger total cross-section than the corresponding arteries. Near to the heart, when distended by the normal pressures, they have about twice the cross-section of the arteries. In other regions the same ratio has been found, and it is said to

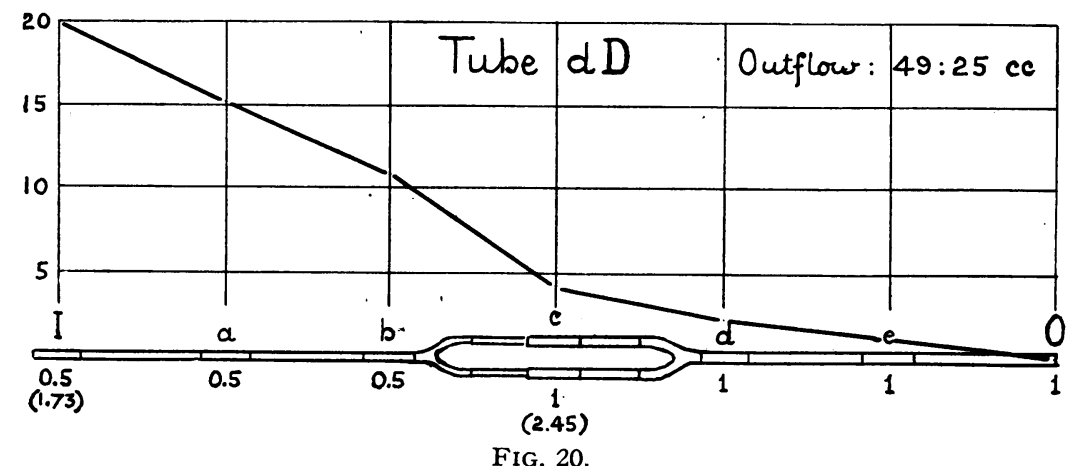

obtain throughout the system ${ }^{4}$. It would be rash to assume that this is strictly true, but let us provisionally assume it to be so and see how this particular asymmetry, apart from any other which 
may be present, would affect the character of the pressure-line. We determined the question by experiment.

In the following tubes the cross-sections at corresponding points in the proximal and distal halves vary as 1 to 2 -with the exception of a few millimetres on each side of the half-way point where they are equal. Sincethe two halves transmit the same amount of water in a given time, the velocity in the proximal half is double that in the distal. What percentage of the total fall of pressure belongs in each case to the distal half ?

In Tube aA (Fig. 14), which presents this asymmetry in its simplest form, a pressure of $20 \mathrm{~cm}$. at $I$ gives $4 \mathrm{~cm}$. at $c$, while $10 \mathrm{~cm}$. at $I$ gives $2 \mathrm{~cm}$. at $c$ : the fall in the distal half is one-fifth, or 20 per cent., of the total fall.

Tube dI (Fig. 20), like the preceding one, was obtained from the corresponding symmetrical tube by reducing the diameter of the proximal half. Here again the distal fall is approximately 20 per cent. (actually 20.5 per cent.) of the total fall.

Tube G G (Fig. 21) was obtained from the symmetrical Tube G by enlarging the distal half instead of by reducing the proximal; we could not in this case reduce the proximal, for the smallest branches of the plexus were already as small as could be satisfactorily employed. The velocity was much greater than in the two previous cases, and the outflows show that the stream was not entirely free from turbulence. The distal fall, nevertheless, is exactly 20 per cent. of the total fall.

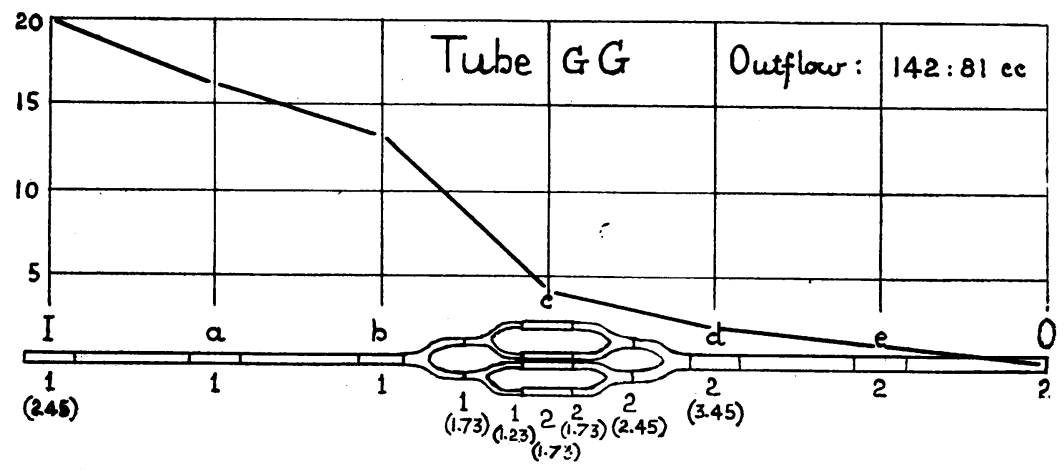

Fig. 21,

Now Tubes A, D, and G, of our symmetrical series, were those in which the cross-section of the channels was uniform throughout : the half-way pressure equalled the mean of the end-pressures; in other words, the distal fall was one-half of the total fall. It is now evident that when such tubes, whether straight or branching, are rendered asymmetrical to the extent in question, the distal fall is reduced from 50 to 20 per cent. of the total fall. 
It remains to ascertain what happens when tubes or systems which enlarge in the middle region, are modified in like manner. We have seen that symmetrical tubes of this kind give a distal fall

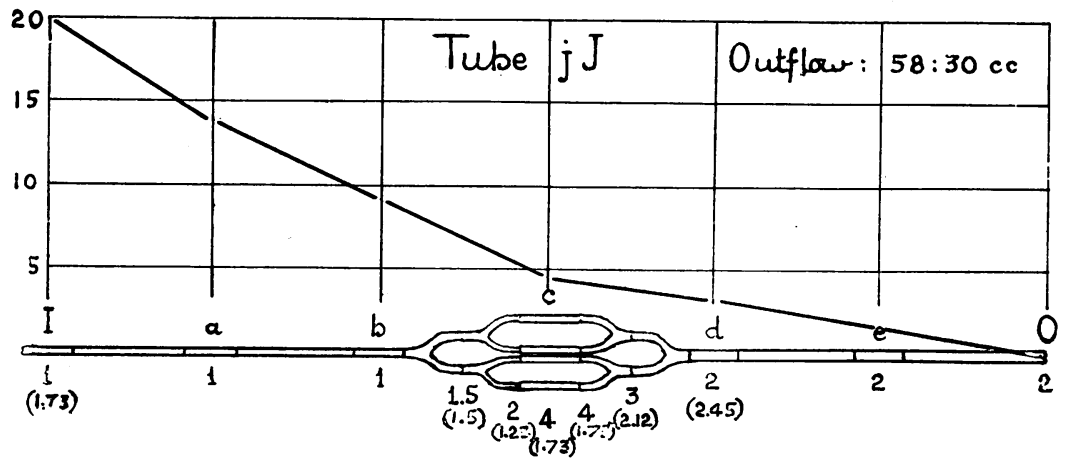

FIG, 22,

in excess of 50 per cent. of the total, and that the excess is smallest when the conditions of velocity, etc., most nearly resemble those of the blood-stream. We may expect that in the corresponding asymmetrical tubes the distal fall will bear a similar relation to 20 per cent. of the total fall; and this proves to be the case.

Tube j J (Fig. 22) shows a distal fall of 22.5 per cent, as compared with 53.5 given by the corresponding symmetrical Tube J.

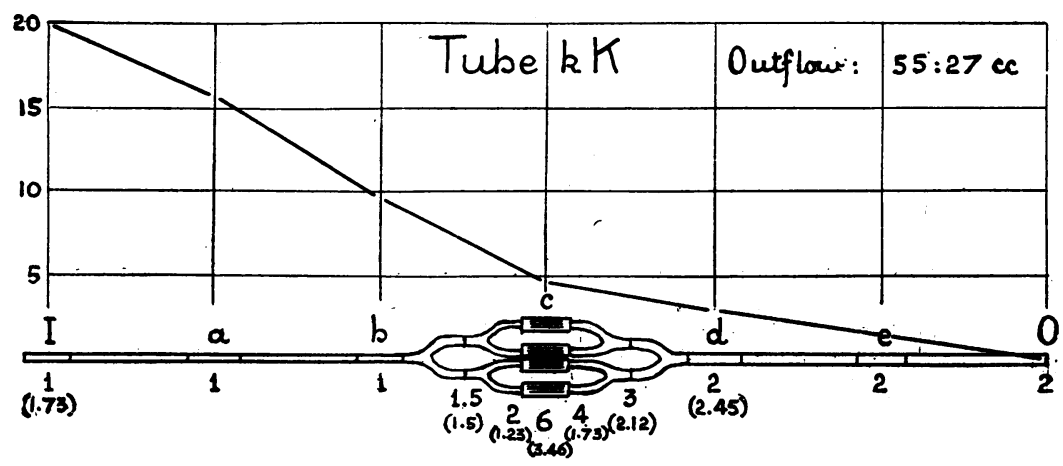

FIG. 23.

Tube k K (Fig. 23) shows 23.5 per cent. as against 55 per cent. given by Tube $\mathrm{K}$.

To modify Tube $\mathrm{O}$ in the same manner was hardly possible, but in view of the foregoing results we may safely assume that since the symmetrical form gives a distal fall of slightly more than 50 per cent., a corresponding asymmetrical tube would give one of slightly more than 20 per cent.

We arrive then at the following conclusion:--If the vascular 
system of the body were horizontal, and if the veins had everywhere twice the cross-section of the corresponding arteries but were equal to them in number and distribution, the fall of pressure in the venous half of the system would be approximately one-fifth of the total fall. A pressure-line obtained from such a system would bear a general resemblance to the lower line in Figure 24. The question is how

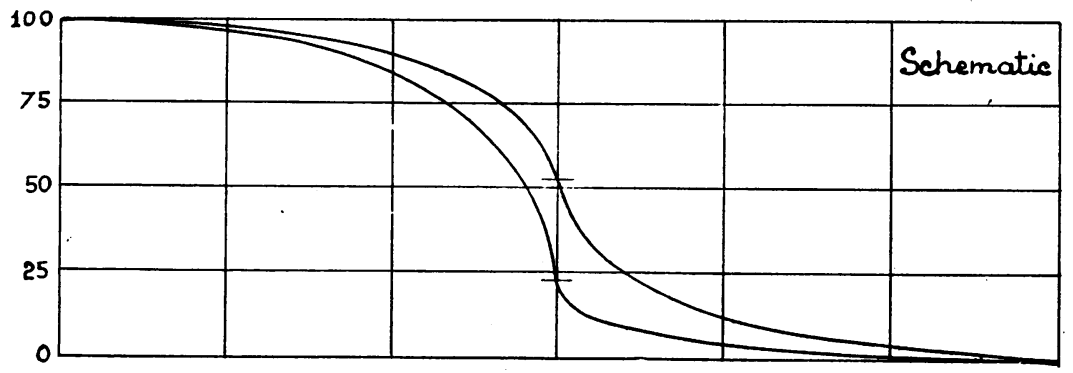

FIG, 24.

far this schematic line differs from the line or lines which the actual blood-stream would give. What sources of fallacy have we disregarded?

In the first place it is clear that no one line can be true for all the different paths that the blood may take in travelling from the left ventricle to the right auricle. At any given time the total fall of pressure will be the same for all paths, because the end pressures are the same, but the length, and consequently the steepness, of the pressure line will vary inversely with the length of the path taken.

In the second place we have calculated the resistances in the two halves of the system on the assumption that the veins are equal to the arteries in number and distribution, whereas in fact, a single artery is often served by two veins or more. Now this multiplying of venous channels must alter the resistance-ratio unless provision to the contrary is made by an alteration of the crosssections. Thus, if the ratio of the cross-sections is everywhere 1 to 2 , the resistances with a single vein will be as 4 to 1 as in Tube aA; with two veins they will be as 2 to 1 as in Tube $R$; with a larger number of veins they would be still nearer to equality, or equal, or even reversed. Veins are multiplied no doubt to safeguard the capillaries against over-distension when one or other vein is obstructed by pressure from without, and it is likely that where they are multiplied they are also increased in aggregate cross-section, so that they shall not offer increased resistance. Otherwise the venous fall in many blood-paths must be much greater than onefifth of the total fall. But we do not know. Therefore our schematic line is open to question as regards the relative amount of pressure at the half-way point. 
In any case this line, and indeed any other which might be devised, can be true only as an average. The pressures in the vascular system of the body change continually under vasomotor influence. Contraction of arterioles lowers the pressure in the corresponding capillaries. Hence, if the pressure in a given capillary area is one-fifth of the initial pressure under ordinary conditions, it will be less than one-fifth when the arterioles feeding that area contract, and more than one-fifth when they dilate.

It is evident that the problems of blood pressure cannot be fully solved by considering, however carefully, the physical principles which are involved. The data at our disposal are insufficient. Yet these principles must be constantly borne in mind when we attempt to appraise the results arrived at in other ways.

REFERENCES.

I. Proceedings of the Royal Society of Medicine, Vol. VI, No. 3, p. 31.

. Nicholls, W.-- Journal of Physiology, Vol. XX, 1896, p. 43.

Vierordt, H. " "Daten und Tabellen," I906, p. I79.

. Starling.- “ Elements of Human Physiology," 8th edition, p. 204.

(To be continued.)

\title{
A CONTRIBUTION TO THE PATHOLOGY OF CHOROIDAL MELANOMATA.
}

\author{
BY \\ R. Foster Moore, F.R.C.S. \\ ASSISTANT SURGEON, ROYAL LONDON OPHTHALMIC HOSPITAL.
}

MELANOMATA of the choroid are of uncommon occurrence, and usually discovered by accident during routine ophthalmoscopic examination.

It is therefore only by some fortuitous chance that a specimen, which has been examined ophthalmoscopically during life, is obtained for microscopic examination.

In Volume XIX, Part iii, of the Royal London Ophthalmic Hospital (Moorfields) Reports were published four cases of this condition which I had examined clinically during life.

All four cases were discovered by accident, and none of them had given rise to symptoms.

A coloured drawing, by A. W. Head, of one of them is given in the Reports, and excellently depicts the ophthalmoscopic characters of these growths.

In one of the cases I was able, owing to the death of the patient from myasthenia gravis, to obtain the specimen, and microphotographs of both bleached and unbleached sections of this tumour are included. 